\title{
Dermatobiosis cutánea bovina en la región selvática lluviosa y montañosa de México
}

\section{Bovine dermatobiosis in rainy forest and mountainous region of Mexico}

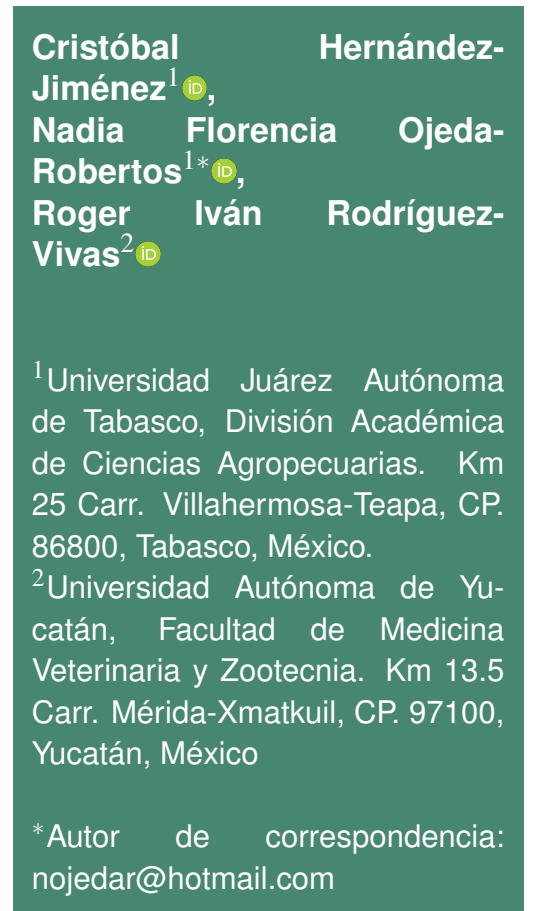

Nota científica

Recibido: 25 de mayo de 2019

Aceptado: 30 de septiembre de 2019

Como citar: Hernández-Jimenez C, Ojeda-Robertos NF, RodríguezVivas RI (2020) Dermatobiosis cutánea bovina en la región selvática lluviosa y montañosa de México. Ecosistemas y Recursos Agropecuarios 7(1): e2303. DOI: 10.19136/era.a7n1.2303
RESUMEN. Se reporta la presencia de Dermatobia hominis en bovinos criados en ranchos ubicados en una zona selvática y montañosa de México. Se realizó un estudio en cuatro ranchos, incluidos por conveniencia. Se incluyeron un total de 75 bovinos, se registró la presencia, el número y características de los nódulos cutáneos por animal, y se colectaron larvas. Las larvas fueron clasificadas taxonómicamente como $D$. hominis. En total $10.6 \%$ de los animales presentaron nódulos en la piel (8/75). Se encontraron entre uno y 22 nódulos por bovino, distribuidos en cuatro regiones corporales (cola, pierna, abdomen y escápula). Se detectó la presencia de larvas $D$. hominis, las miasis que producen se caracterizan por la presencia de nódulos en la escápula, cola y pierna. Se recomienda que en la zona de estudio se implementen medidas de control de la mosca a través del uso de antiparasitarios en los bovinos.

Palabras clave: Dematobia hominis, infestación, prevalencia, miasis, nódulos cutáneos.

ABSTRACT. The presence of Dermatobia hominis in cattle raised in a forest and mountainous area of Mexico is reported. A study was conducted on four farms, included for convenience. A total of 75 cattle were included, the presence, number and characteristics of skin nodules per animal were recorded, and larvae were collected. The larvae were taxonomically classified as $D$. hominis. In total, $10.6 \%$ of the animals presented nodules on the skin (8/75). They were found between one to 22 nodules per bovine, distributed in four body regions (tail, leg, abdomen and scapula). It is concluded that, the presence of $D$. hominis larvae was detected and that the myiases they produce are characterized by the presence of nodules in the scapula, tail and leg. It is recommended that fly control measures be implemented in the study area through the use of antiparasitics in cattle.

Key words: Dermatobia hominis, infestation, prevalence, miasis, cutaneous nodules. 


\section{INTRODUCCIÓN}

La dermatobiosis cutánea bovina (DCB), es una parasitosis producida por la mosca Dermatobia hominis (Diptera: Cuterebridae), la cual en su estadio larvario afecta el tejido subcutáneo del huésped. La característica principal de la dermatobiosis parasitaria es que se produce una miasis furuncular, con inflamación, secreción de líquidos y lesiones en la piel que son dolorosas para el animal, lo que ocasiona intranquilidad a los animales afectados y pérdida de peso, además de que representan una fuente de infección o reservorio para el ser humano (Reina y Villarreal 2017, Failoc et al. 2018). El impacto económico de la DCB es por las pérdidas que ocasiona en la producción de leche, carne y piel (Souza et al. 2010). En Brasil, se ha estimado que una sola larva de $D$. hominis en un bovino produce una reducción de la ganancia de peso de 40.6 g (Magalhães y Lesskiu 1982). En otros estudios se ha estimado que las pérdidas directas que produce a la ganadería bovina de Brasil es de 383.48 millones de dólares americanos al año (Grisi et al. 2014). También se reportan pérdidas económicas por el decremento en la calidad de la piel debido a las perforaciones que ocasionan las larvas del parásito (Zammarchi et al. 2014).

El ciclo de vida del díptero inicia con la cópula y ovoposición de los huevos, una hembra fértil de $D$. hominis deposita de 5 a 30 huevos sobre el cuerpo de otros insectos que actúan como vectores mecánicos o foréticos (Alencar et al. 2010). Se reporta que existen varias especies de artrópodos pertenecientes a las familias Muscidae, Anthomyiidae, Tabanidae, Sarcophagidae, Simulidae, Culicidae y Cuterebridae que son utilizados por la mosca hembra para el transporte (Robbins y Khachemoune 2010). Pero, las especies Haematobia irritans, Musca domestica y Stomoxys calcitrans son las más importantes en el transporte de huevos de la mosca $D$. hominis (Alencar et al. 2010, Robbins y Khachemoune 2010). Cuando la mosca forética se posa sobre el bovino, el calor emanado del animal estimula la eclosión del huevo y sale la larva $\left(L_{1}\right)$, la cual penetra la piel con la ayuda de sus ganchos bucales, hasta larvas $L_{2}$ y $L_{3}$, y es durante este periodo, que se desarrollan nódulos ó forúnculos dolorosos para el animal. La larva $\mathrm{L}_{3}$ abandona al huésped a través de un orificio de la piel lesionada, y cae al suelo, se entierra e inicia la formación de la pupa (Cardona et al. 2013). Después de este período se produce un imago o mosca joven, que en pocas horas es fecundada para iniciar el ciclo de vida. El período pupal es crítico y su duración varía dependiendo de las condiciones climáticas principalmente de la temperatura (Mateus y Cadena 1973, Cardona et al. 2013). Dermatobia hominis y la enfermedad que produce (DCB) es endémica en México y con amplia distribución geográfica entre los $24^{\circ}$ y $26^{\circ}$ latitud Norte, América Central y Sudamérica, exceptuando Chile y algunos distritos de Argentina y ocurre predominantemente en climas templados, aunque también se puede encontrar en regiones montañosas (Francesconi y Lupi 2012, Villalobos et al. 2016).

En México, la DCB es llamada comúnmente "colmoyote", con casos en humanos, animales domésticos y silvestres en la región sureste del país (Thomas 1987). También se reporta que muchas especies de animales albergan la fase larvaria de esta mosca, entre las que destacan: bovinos, ovinos, cerdos, caballos, perros, venados cola blanca (Odocoileus virginianus), pecarís de collar (Tayassu tajacu), mapaches (Procyon lotor) y jaguares (Panthera onca). La enfermedad en bovinos en Brasil se ha reportado en $40 \%$ de ranchos con sistema de producción intensivo. (Mozzaquatro y Sanavria 2003, Ferraz et al. 2014). Por otra parte, en humanos, existen reportes de $D$. hominis en viajeros que han visitado regiones tropicales de Latinoamérica y que al regresar desarrollaron los signos clásicos de la presencia de la larva (Zammarchi et al. 2014). En México se tienen casos esporádicos, para el estado de Quintana Roo se reporta un caso de dermatitis nodular en una persona que visitó los estados de Quintana Roo y Chiapas (Rodríguez y Aquino 2009). En los últimos años, se ha incrementado los casos en regiones tropicales y selváticas de México, sin conocerse, hasta el momento, la prevalencia de la parasitosis en zonas endémicas así como su distribución y efecto en la salud de los bovinos. Por tal 
motivo, el objetivo fue determinar la dermatobiosis cutánea bovina producida por larvas de $D$. hominis en bovinos criados en ranchos de una zona selvática y montañosa de México.

\section{MATERIALES Y MÉTODOS}

\section{Ubicación del área de estudio}

El área de estudio se encuentra localizada entre los $17^{\circ} 07^{\prime \prime} \mathrm{LN}$ y $92^{\circ} 17^{\prime \prime}$ LO. Los ranchos estudiados pertenecen al municipio de Chilón, ubicado en la zona VI Selva al norte del estado de Chiapas, México (Figura 1). El municipio posee un clima cálido a semi-cálido húmedo $(\mathrm{A}) \mathrm{C}(\mathrm{m})$, con altitud promedio de $1000 \mathrm{msnm}$, precipitación pluvial anual de $3087 \mathrm{~mm}$ y temperatura media anual de $24^{\circ} \mathrm{C}$. Los suelos de la región se clasifican como Regosoles y la orografía de la región es mixta con predominancia de zonas accidentadas. Los tipos de vegetación corresponden a bosque mesófilo de montaña y selva mediana perennifolia (INEGI 2017). Los ranchos ganaderos estudiados se encuentran ubicados a 6 $\mathrm{km}$ de la cabecera municipal, con una distancia entre ellos de $2 \mathrm{~km}$ aproximadamente, limitan entre si y están divididos por mallas de alambre. El acceso a los ranchos es limitado y únicamente es posible entrar caminando.

\section{Ranchos de estudio}

Se realizó un estudio descriptivo observacional donde se incluyeron cuatro ranchos productores de bovinos, los cuales se incluyeron por conveniencia y por las facilidades de los propietarios para realizar el trabajo. Además de que tienen la presencia de bovinos con nódulos en la piel y tejido subcutáneo. Las unidades se dedican a la producción de carne bovina, tres con sistemas de producción extensiva y uno semiextensiva. Las unidades cuentan con poblaciones de entre 8 y 30 bovinos de las cruzas de razas europeas con cebuínas, en diferentes estadios fisiológicos. Los animales son criados con pastoreo de pastos introducidos como estrella de áfrica (Cynodon nlemfluensis) y grama nativa (Paspalum notatum y $P$. conjugatum).

\section{Diseño del trabajo}

Se visitaron cuatro ranchos en abril de 2018 (época de seca) para realizar una inspección en 75 animales y realizar la colecta de muestras. Adicionalmente, se realizó una entrevista al propietario de cada rancho con el fin de conocer los antecedentes y manejo zootécnico general. Se puso especial énfasis en los detalles clínicos de los animales con lesiones en la piel y presentación de la infección de larvas de D. hominis en los bovinos.

\section{Obtención de muestras}

Todos los bovinos se inspeccionaron de forma individual, para lo cual, cada animal se sujetó para realizar palpación minuciosa desde la cabeza a la cola en busca de nódulos y lesiones en la piel. Los animales en los cuales se detectó la presencia de al menos un nódulo se consideraron positivos, adicionalmente se registró el cuerpo y el número de nódulos de cada animal. Los nódulos se midieron con una regla graduada en $\mathrm{cm}$ para determinar su tamaño.

\section{Identificación de especímenes}

Se determinó por medio de la palpación la factibilidad de liberar los parásitos presentes en los nódulos por medio de compresión manual. De cada nódulo y en la medida de lo posible se extrajeron los parásitos de forma que no resultaran dañados por la manipulación, por lo que en algunos de ellos fue necesario realizar ligera tracción con los dedos. Una vez colectadas las larvas, se identificaron y conservaron hasta su trasladado al laboratorio de Parasitología Animal de Centro de Investigación en Ciencias Agropecuarias de la División Académica en Ciencias Agropecuarias de la Universidad Juárez Autónoma de Tabasco, donde los especímenes colectados en campo, se lavaron con agua estéril para retirar secreciones, restos de pelo y conservarlos en viales con alcohol al $70 \%$ hasta su identificación. Posteriormente, las muestras se enviaron al laboratorio de Parasitología del Campus de Ciencias Biológicas y Agropecuarias de la Universidad Autónoma de Yucatán para su identificación con las claves taxonómicas de Thanapatcharoen et al. 


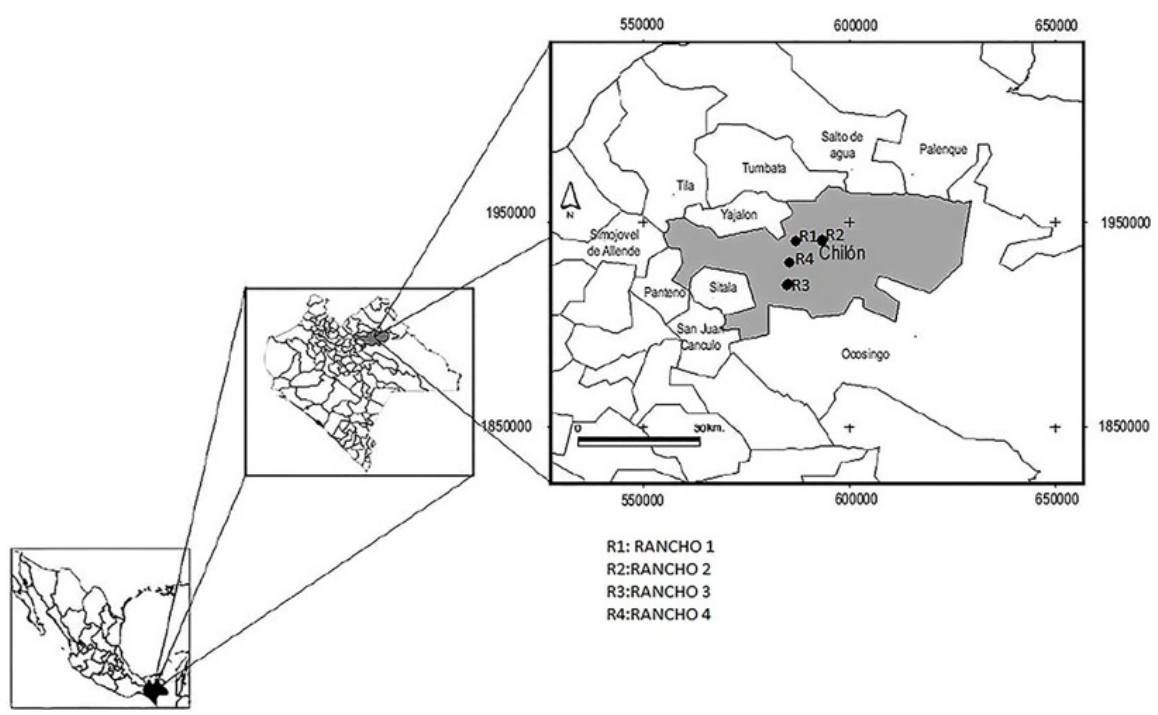

Figura 1. Localización de los cuatro ranchos en el municipio de Chilón, Chiapas, México, donde se realizó el estudio de larvas de Dermatobia hominis en bovinos, modificado de INEGI (2017).

(2012) y West (2013)

\section{Análisis de datos}

Con el propósito de conocer la asociación de la edad con la presentación de las infecciones por $D$. hominis, la edad de los bovinos se clasificó en lactantes-jóvenes (2-7 meses de edad) y destetesadultos ( $>7$ meses de edad). Para lo cual se realizó una prueba de $X^{2}$ a través de un cuadro de contingencia $2 \times 2$. Por tener valores menores de 5 , se usó la prueba de corrección de Yates con un nivel de significancia $\mathrm{P}<0.05$, con el programa estadístico Statistix versión 9 .

\section{RESULTADOS Y DISCUSIÓN}

Las larvas colectadas se clasificaron taxonómicamente como $D$. hominis (Figura 2), dicho parásito ha sido reportado parasitando animales domésticos, silvestres y humanos en el sureste de México (Rodríguez y Aquino 2009). Pero hasta donde se sabe no existen datos actualizados de la prevalencia del parásito en bovinos de la zona sur de México, ya que la literatura se centra más en la descripción de la enfermedad de casos aislados en seres humanos que han sido parasitados y diagnosticados con miasis producida por larvas de $D$. hominis (Zuñiga 2009).
La prevalencia general de lesiones en la piel causadas por larvas de $D$. hominis en los bovinos de los cuatro ranchos estudiados fue de $10.6 \%(8 / 75)$, presentándose en los ranchos prevalencias de $3.8 \%$ a $18.0 \%$ (Tabla 1). La presencia de esta miasis está influenciada por el área geográfica (altitudes de 600 a $1400 \mathrm{msnm}$ ) y la presencia de alta precipitación pluvial (Ferraz et al. 2014); como la zona donde se realizó el presente estudio, la cual se ubica a 880 msnm (Reina y Villarreal 2017). Debido a que solo se realizó durante el mes de abril, es necesario continuar con estudios durante todo el año para establecer los periodos de aumento de casos a lo largo del año. De los ocho bovinos positivos con lesiones por larvas de $D$. hominis, el $16.6 \%(5 / 30)$ fueron animales lactantes-jóvenes y $6.6 \%(3 / 45)$ animales destetesadultos, no habiendo diferencias significativas entre las edades estudiadas $(p=0.32)$. Lo que coincide con Reina y Villarreal (2017) quienes mencionan que no existe preferencia de la mosca por la edad de los animales.

La lesiones o nódulos se ubicaron en cola, pierna, abdomen y escápula (Tabla 1, Figura 3), lo que coincide con lo reportado en la literatura, ya que se sugiere que las áreas corporales más infestadas por $D$. hominis en bovinos son los miembros anteriores, el tórax y la escápula, con frecuencias de 


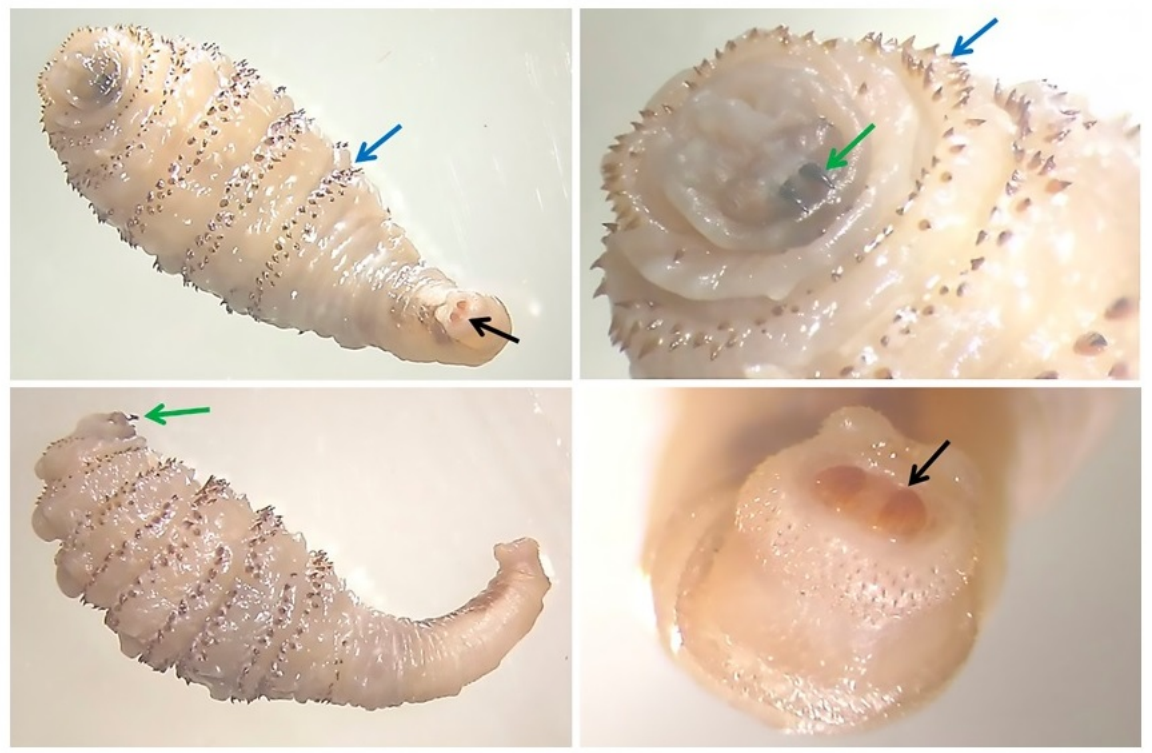

Figura 2. Larva $\mathrm{L}_{3}$ de Dermatobia hominis. a) Filas paralelas concéntricas de espinas (flechas azules), b) ganchos bucales (flechas verdes) y c) abertura de respiración o espiráculo (flecha negra).

Tabla 1. Número de bovinos, edad, localización de nódulos cutáneos conteniendo larvas de Dermatobia hominis en cuatro ranchos de Chilón, Chiapas, México.

\begin{tabular}{|c|c|c|c|c|c|}
\hline Rancho & $\begin{array}{c}\text { Bovinos positivos/ } \\
\text { Población } \\
\text { (Prevalencia) }\end{array}$ & Edad & $\begin{array}{l}\text { Localización } \\
\text { de } \\
\text { nódulos }\end{array}$ & $\begin{array}{l}\text { Número de nódulos } \\
\text { (número de larvas } \\
\text { en nódulos) }\end{array}$ & Signos clínicos \\
\hline 1 & $\begin{array}{c}2 / 11 \\
(18.1 \%)\end{array}$ & $\begin{array}{l}2 \text { meses } \\
1.4 \text { años }\end{array}$ & $\begin{array}{l}\text { Cola y pierna } \\
\text { Escápula }\end{array}$ & $\begin{array}{l}2(1-2) \\
5(1-2)\end{array}$ & $\begin{array}{c}\text { Dolor al presionar los nódulos, expulsión } \\
\text { de material purulento y sangre. }\end{array}$ \\
\hline 2 & $\begin{array}{c}1 / 8 \\
(12.5 \%)\end{array}$ & 7 años & Escápula & $22(2-3)$ & $\begin{array}{l}\text { Dolor al presionar } \\
\text { los nódulos. }\end{array}$ \\
\hline 3 & $\begin{array}{c}4 / 30 \\
(13.3 \%)\end{array}$ & $\begin{array}{l}4 \text { meses } \\
6 \text { meses } \\
7 \text { meses } \\
6 \text { años }\end{array}$ & $\begin{array}{l}\text { Cola y dorso } \\
\text { Cola } \\
\text { Dorso y escápula } \\
\text { Abdomen }\end{array}$ & $\begin{array}{c}4(1-2) \\
2(1-2) \\
5(1-3) \\
1(4)\end{array}$ & $\begin{array}{c}\text { Dolor al } \\
\text { presionar los nódulos } \\
\text { expulsión de } \\
\text { material purulento. }\end{array}$ \\
\hline $\begin{array}{l}4 \\
\text { Prevalen }\end{array}$ & $\begin{array}{c}1 / 26 \\
(3.8 \%) \\
\text { General } 8 / 75(10.6 \%)\end{array}$ & 4 meses & Cola & $1(4)$ & $\begin{array}{l}\text { Alopecia en el } \\
\text { área del nódulo. }\end{array}$ \\
\hline
\end{tabular}

$34.5 \%, 27.8 \%$ y $13.5 \%$, respectivamente (Mozzaquatro y Sanavria 2003). Po lo que la ubicación de estas lesiones, corresponden a las áreas que normalmente se ven parasitadas por los artrópodos hematófagos. El número de nódulos en los bovinos varió de 1 a 22 (Tabla 1). En cada nódulo se extrajeron de uno a cuatro larvas, con diferentes estados de desarrollo $\left(L_{1}, L_{2}\right.$ y $\left.L_{3}\right)$. Lo que pone de manifiesto el potencial de la infección de las larvas de $D$. hominis y su capacidad para producir lesiones en la piel de los animales estudiados. De acuerdo con Cardona et al. (2013) la presencia de 20 a 40 nódulos origina en los animales una disminución del $12 \%$ en el peso del animal $y$ en el ganado lechero produce una merma del $20 \%$ en la producción de leche. A las pérdidas señaladas se le debe agregar el gasto que origina la compra de medicamentos y la mano de obra necesaria para tratar a los animales afectados. Por lo que se requiere que en futuros estudios se determine el efecto negativo de las infecciones de larvas de $D$. hominis en el ganado bovino de la región. Para poder controlar y reducir el riesgo de infección de bovinos con larvas 


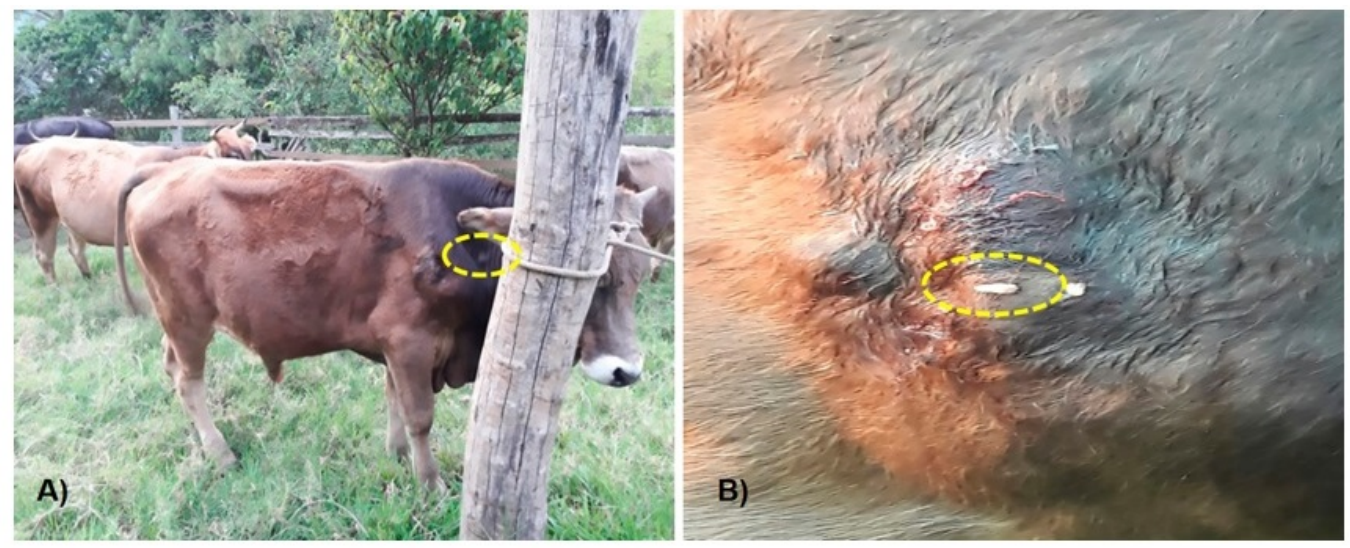

Figura 3. Nódulos en un bovino producido por la presencia de diferentes estadios larvarios del díptero Dermatobia hominis. A) nódulos en la región de la escápula y en el cuello, B) nódulo con una larva de $D$. hominis emergiendo.

de $D$. hominis y su posible infección a la población humana en la zona sur de México, se recomienda implementar medidas de control de la mosca durante su mayor actividad (épocas de lluvia y alta humedad) a través del uso de drogas antiparasitarias en animales tales como lactonas macrocíclicas (Reina y Villarreal 2017).

Se detectó la presencia de larvas $D$. hominis y las miasis que producen se caracterizan por la presencia de nódulos en la escápula, cola y pierna. Se recomienda que en la zona de estudio se implementen medidas de control estratégico de la mosca durante su mayor actividad a través del uso de antiparasitarios en los bovinos.

\section{AGRADECIMIENTOS}

A los propietarios de los ranchos por la confianza y facilidades proporcionadas en la realización del estudio. Al EMVZ de la DACA-UJAT Lizz Nayeli Hernández-Martínez y al Sr. Humberto Reyes Díaz, por la incondicional ayuda otorgada durante la fase de campo para la colecta de los especímenes. A los laboratorios de Parasitología de la División Académica de Ciencias Agropecuarias de la UJAT y de la Universidad Autónoma de Yucatán por el apoyo y facilidades para el procesamiento de las muestras.

\section{LITERATURA CITADA}

Alencar RB, Saraiva JF, Oliveira AFJ, Scarpassa VM (2017) First record of Anopheles konderi Galvão \& Damasceno (Diptera: Culicidae) carrying eggs of Dermatobia hominis (Linnaeus Jr.) (Diptera: Oestridae), from Oriximiná municipality, Pará, Brazil. Revista da Sociedade Brasileira de Medicina Tropical 50: 388-390.

Cardona AJA, Montes VJC, Castaño VFA, Blanco MRD, Gómez LVE (2013) Frecuencia de dermatobiosis cutánea bovina en vacas Holstein de un hato lechero en Viçosa (MG, Brasil). CES Medicina Veterinaria 8: 82-84.

Failoc R, Molina A, Salazar Z, Samamé A, Silva D (2018) Case Report: Myiasis due to Cochliomyia hominivorax and Dermatobia hominis: clinical and pathological differences between two species in Northern Peru. The American Journal of Tropical Medicine and Hygiene 98: 150-153.

Ferraz da Costa M do S, Guimarães MP, Lima Wdos S, Ferraz da Costa AJ, Facury Filho EJ, Araujo RN (2014) Seasonal variation and frequency distribution of ectoparasites in crossbreed cattle in Southeastern Brazil. Journal of Veterinary Medicine. Article ID 759854. Doi: 10.1155/2014/759854.

Francesconi F, Lupi O (2012) Myiasis. Clinical Microbiology Reviews 25: 79-105. 
Grisi L, Cerqueira Leite R, Martins JR, Barros AT, Andreotti R, Cançado PH, León AA, Pereira JB, Villela HS (2014) Reassessment of the potential economic impact of cattle parasites in Brazil. Revista Brasileira de Parasitology Veterinary 3: 150-156.

INEGI (2017) Instituto Nacional de Estadística y Geografía. Anuario estadístico y geográfico de Chiapas. http:// www.inegi.org.mx/est/contenidos/espanol/sistemas/aee12/estatal/chis/default.htm. Fecha de consulta: 22 de Julio de 2017.

Magalhães FEP, Lesskiu C (1982) Efeito do controle do berne sobre o ganho de peso e qualidade dos couros em novilhos de corte. Pesquisa Agropecuaria Brasileira 17: 329-336.

Mateus VG, Cadena SJA (1973) El nuche: vida y control. ICA informa BAC Modulo Digital 8: 5-12.

Mozzaquatro F, Sanavria A (2003) Estudo epidemiológico da Dermatobia hominis (Diptera: Cuterebridae) em bovinos de produção leiteira no Município de Santa Maria, Rio Grande do Sul, Brasil. Parasitología Latinoamericana 58: 80-82.

Reina I, Villarreal B (2017) Dermatobia hominis: larvas destructoras escondidas bajo la piel de nuestros bovinos. Actualidad Ganadera 4: 26-30.

Robbins K, Khachemoune A (2010) Cutaneous myiasis: a review of the common types of myiasis. International Journal of Dermatology 49: 1092-1098.

Rodríguez AM, Aquino PG (2009) Miasis furunculoide múltiple. Revista del Centro Dermatológico Pascua 18: 96-99.

Souza FS, Botelho MCSN, Lisbôa RS (2010) Caracterização de associação entre a sazonalidade de larvas de Dermatobia hominis em bovinos, dípteros potenciais vetores e dados meteorológicos de três diferentes locais no Rio de Janeiro. Revista Brasileira de Ciencia Veterinaria 17: 111-116.

Thanapatcharoen A, Preativatanyou K, Phumee A, Kraivichain K (2012) Cutaneous myiasis caused by Dermatobia hominis in Thai travelers: First report in Thailand. Asian Biomedicine 6: 487-494.

Thomas DB (1987) Incidence of screwworm (Diptera: Calliphoridae) and torsalo (Diptera: Cuterebridae) myiasis on the Yucatan Peninsula of Mexico. Journal of Medical Entomology 24: 498-502.

Villalobos G, Vega-Memije ME, Maravilla P, Martinez-Hernandez F (2016) Myiasis caused by Dermatobia hominis: countries with increased risk for travelers going to neotropic areas. International Journal of Dermatology 55: $1060-8$.

West J (2013) Simple and effective field extraction of human botfly, Dermatobia hominis, using a venom extractor. Wilderness \& Environmental Medicine 24: 17-22.

Zammarchi L, Viligiardi R, Strohmeyer M, Bartoloni A (2014) Dermatobia hominis: Small migrants hidden in your skin. Annals of Dermatology 26: 632-635.

Zuñiga CIR (2009) Miasis un problema de salud poco estudiado en México. Revista de Enfermedades Infecciosas en Pediatría 22: 121-125. 